\title{
LA WEB 2.0 HERRAMIENTA DE EMPODERAMIENTO DE LAS EMPRESARIAS PYMES. UN ESTUDIO DE CASO EN EL CONTEXTO ANDALUZ
}

\author{
WEB 2.0 TOOL OF EMPOWERMENT OF FEMALE \\ ENTREPRENEURS SMES. A CASE STUDY IN \\ THE ANDALUSIAN CONTEXT
}

\author{
Rosario RODRÍGUEZ-DÍAZ \\ Universidad de Sevilla \\ María-José GONZÁLEZ-Río \\ Universidad de Alicante
}

Recibido:03/11/2015

Aceptado:09/05/2016

\section{Resumen}

El principal objetivo de esta investigación es identificar a través del Modelo de Aceptación Tecnológica (TAM) los factores que impulsan los usos, grado de inmersión y utilidades de las redes sociales, así como las potenciales motivaciones o barreras en microempresas y/o pymes dirigidas por mujeres en la Comunidad de Andalucía. Se trata de un estudio cualitativo realizado a través de entrevistas en profundidad de 14 empresarias elegidas mediante muestreo no probabilístico -casos con máxima variación- al objeto de obtener un mayor espectro representativo de experiencias de uso y de perfiles sociodemográficos. El empresariado femenino estudiado no presenta una afectación homogénea en el nivel de uso de los social media como herramienta de sus negocios, sin embargo son conscientes de su eficacia. Su competitividad futura va a estar altamente supeditada por la utilización eficiente de las redes sociales virtuales que la nueva economía digital demanda.

Palabras clave: emprendimiento digital femenino, Social media, Modelo de Aceptación Tecnológica (TAM), Empresarias de pymes, pymes 2.0. 


\begin{abstract}
The main objective of this research is to identify through the Technology Acceptance Model (TAM) factors that drive uses, level of immersion and utilities the social media as well as potential motivations or barriers in micro and / or SMEs run by women in the region of Andalusia. This is a qualitative study through interviews in depth of 14 female entrepreneurs selected by non-probability sample and maximum variation sampling, in order to get a more representative spectrum of experiences of use and socio-demographic profiles. Female entrepreneurship has not studied a homogeneous level of involvement in the use of social media as a tool for their business, but they are aware of their effectiveness. Its future competitiveness will be highly contingent on the efficient use of virtual social networks that demand the new digital economy.
\end{abstract}

Keywords: Digital Female Entrepreneurship, Social media, Technology Acceptance model (TAM), Women micro entrepreneurs, 2.0 SMEs. 


\section{INTRODUCCIÓN}

La vicepresidenta de la Comisión Europea, Neelie Kroes, en el año 2010 desveló la iniciativa Digital Agenda For Europe -ADE- cuyo objetivo suponía trazar un plan que materializase plenamente el potencial del futuro digital de Europa. Con ello se inicia la configuración de una sociedad digital que deberá conseguir un crecimiento sostenible e inclusivo en el horizonte 2020.

Al amparo de las directrices de la Unión Europea, España aprueba en el año 2013 su proyecto en materia de políticas públicas en Tecnologías de la Información y Comunicación (en adelante TIC), condensado en la Agenda Digital para España. Su principal objetivo es provocar una transformación y modernización del conjunto de la sociedad y economía digital a través de un uso eficaz e intenso de las TIC por parte de su ciudadanía, del tejido empresarial y de la administración en su conjunto. En el ámbito empresarial que nos ocupa, se trata de «La utilización eficiente e intensiva de las TIC en las empresas, especialmente en las pymes y microempresas, como factor imprescindible para mejorar la productividad de nuestra economía» (Agenda Digital para España 5).

Es evidente que en este esfuerzo se abren oportunidades para generar, distribuir riqueza y conocimiento, al diversificar y flexibilizar los usos de tiempo y espacio en el avance hacia una sociedad más incluyente y equitativa. Pero, en su anverso, esta vertiginosa permeabilidad digital no deja de estar exenta de fracturas y segregaciones que alertan ante este nuevo panorama. Un ejemplo de ello es la existencia de brechas digitales discriminatorias que afectan a colectivos, ciudadanos y empresas (DiMaggio, Hargittai, Celeste, Shafer 356358; Hargittai, Walejko 240-242; Livingstone, Helsper 673-675; Norris 4). De manera global, hombres y mujeres se enfrentan a posiciones asimétricas ante esta realidad económico-empresarial ${ }^{1}$. Precisamente, la Agenda Digital para España contempla en su línea 6 (Inclusión y alfabetización), del Plan de

1. Los emprendedores en su mayoría son hombres. El 63\% son autónomos hombres frente al $37 \%$ de mujeres y el $70 \%$ de empresarios son hombres frente al 30\% de mujeres. Cuánto más grande es la empresa más aumenta la diferencia entre hombres y mujeres. En empresas de 1 a 5 empleados el $69 \%$ de los emprendedores son hombres y el 
Acción para la Igualdad de Mujeres y Hombres en la Sociedad de la Información, «La promoción del empresariado TIC femenino» (50-51).

La Web 2.0 es una tecnología con un profundo potencial de cambio. A través de ella se están consiguiendo modos de comunicación más eficientes entre las empresas,-sus interlocutores -tanto internamente, como a nivel de mercado- y con sus entornos. Para cualquier empresa las comunidades virtuales o redes sociales virtuales (en adelante RSV) son una herramienta esencial y una oportunidad para generar imagen de marca y convertir seguidores en clientes (Fundación Banesto 49). En España, pese a situarse en los primeros puestos en uso y tiempo en la Web 2.0, aún queda un largo recorrido a nivel empresarial en cuanto a la adopción de tecnologías sociales por sectores, regiones y género.

El artículo presenta los hallazgos obtenidos a partir de las entrevistas realizadas a una muestra de empresarias en Andalucía. El análisis de sus opiniones nos ha permitido recabar información sobre los usos, grado de inmersión, motivaciones y dificultades en el empleo de los social media en las pymes andaluzas. La realización de este trabajo empírico permite poner nuestro "granito de arena» en la apertura de una línea novedosa de investigación sobre el proceso de cambio que está llevando a cabo el empresariado femenino. Consideramos, además, que el empleo de la metodología cualitativa es otra de las aportaciones principales de nuestra investigación.

El presente documento se articula de la manera siguiente: iniciamos nuestra singladura con el planteamiento del marco teórico en el que se fundamenta la investigación. El trabajo continúa con la exposición metodológica y, el análisis e interpretación de resultados. En el último apartado, y a modo de epílogo, se abordan las conclusiones más significativas del estudio así como sus limitaciones y posibles líneas futuras.

\section{MODELOS TEÓRICOS. TECHNOLOGY ACCEPTANCE MODEL (TAM)}

La plena actualidad de la economía digital que atrae la atención de las directrices político-institucionales de profundo calado, contrasta con la falta de estudios específicos desagregados por género en el mundo empresarial. En lo referente a bibliografía e investigaciones que combinen las temáticas

31\% mujeres, según datos del Observatorio de Clima Emprendedor 2012 (Fundación Iniciador-Sage 10).

Según la Asociación de Trabajadores Autónomos (ATA), Federación de Autónomos, en su Informe Mujer Autónoma 2015, la cifra de mujeres autónomas estaría en el 35,2\%, frente al $64,8 \%$ de varones. 
empresariado femenino y el uso de los social media, nos encontramos ante un campo cuasi yermo en nuestro país (Rodríguez-Díaz, González-Río, RebolloCatalán 13) y no muy prolijo a nivel internacional, pese a atisbarse sus beneficios (Lugaye, Slabbeert, Ukpere 553)

Esta investigación responde a la necesidad de segmentar las relaciones entre las comunidades virtuales y el género. Éstas no se producen en vacío, sino en conjunción con otras variables: económicas, profesionales, personales, sociales, educativas, etc., que definen y enmarcan la vida real. En efecto, variables como la edad, el nivel educativo y la profesión, amén de los conocimientos, experiencias e intereses, son aspectos a sopesar a la hora de analizar las brechas digitales de género (Brynin 440-441; Korupp, Szydlik 410-412; Losh). Es decir, la «definición social» (Torres, Robles, Molina 372) que configura estos colectivos y situaciones puede actuar de manera propiciadora o adversa para el uso de estas herramientas por parte del empresariado femenino.

Nuestro trabajo se sustenta en el conocido modelo Technology Acceptance Model (TAM), de Freed D. Davis (1989) que identifica los factores que impulsan la adopción y uso de las tecnologías. Davis postuló que las personas usuarias de tecnologías dependían de la facilidad de uso percibido-Perceived Ease of use- (PEoU) y de la percepción de utilidad -Perceived Usefulness(PU), es decir, que los individuos emplearían las TIC con más frecuencia en la medida en que las percibiesen fáciles de usar y útiles en sus resultados. Mientras la facilidad atañe al grado que una persona cree que el uso de una determinada tecnología estará libre de esfuerzo, la utilidad alude a la percepción subjetiva de que el uso de un determinado sistema mejorará su actuación en el trabajo. El TAM posiciona estos dos conceptos en el núcleo de su modelo. No obstante, incorpora al mismo ciertos aspectos de la Teoría de la Acción Razonada -Theory of Reasoned Action (TRA)- de Fishbein y Azjen entre los que se encuentra la intención personal en la determinación de si ocurrirá o no un comportamiento determinado. En efecto, a la percepción de facilidad de uso (PEoU) y a la percepción de utilidad (PU), se le añade un concepto objetivo y racional de la teoría (TRA): la intención conductual (Davis 1986, 35-47).

Tras la validación de las mediciones del TAM se ha ido produciendo una extensión del modelo al identificar variables externas que podían influir en el PU y PEoU (Lee, Kozar, Larsen), incluyendo características individuales, organizacionales y de tareas. Con ello se ha pasado de tener una orientación psicológica a otra más social. Venkatesh y Davis propusieron una versión extendida de TAM, denominado TAM2 en el que se incluyeron un grupo de 
variables determinantes y moderadoras de PU (Venkatesh, Davis). Pero, más tarde Venkatesh y sus colaboradores propusieron el Unified Theory of Acceptance and Use of Technology (UTAUT), configurada por otros modelos (ocho, incluido el TAM) que competían una explicación sobre el porqué las personas adoptan y usan tecnologías (Venkatesh, Morris, Davis, Davis).

Los conceptos de facilidad de uso y utilidad son manejados en prácticamente la totalidad. Según la teoría unificada (UTAUT), el uso de la tecnología depende de la intención de comportamiento, condicionada por variables como la expectativa de rendimiento, la expectativa del esfuerzo, la influencia social y las condiciones que la facilitan. Los autores también consideran que género, edad, experiencia y voluntad son variables moderadoras de las variables anteriores y de la intención de comportamiento. Años más tarde Venkatesh y Bala anunciaron una última extensión del modelo TAM3, en el que añaden algunas variables determinantes de PEoU. En general, las diferentes versiones y ampliaciones proponen factores moderadores que pueden influir en la relación de los constructos y la variabilidad explicada por el modelo.

Pese a la evolución que ha experimentado el modelo TAM, sigue estando constituido por un conjunto simple de variables y continúa siendo un gran modelo para indagar por qué las personas adoptan tecnologías. Entre sus limitaciones se mencionan el carácter cuantitativo que han tenido los estudios relacionados con el modelo, de ahí la necesidad de realizar investigaciones tecnológicas con paradigmas más interpretativistas (Lee et al. 769).

\section{OBJETIVOS Y METODOLOGÍA}

Presentamos los resultados de una investigación efectuada entre octubre de 2014 y mayo de 2015. Su finalidad estriba en conocer el grado de implantación, usos y motivaciones de las nuevas redes de comunicación en pymes y microempresas dirigidas por mujeres en Andalucía. Sus discursos recogen experiencias condicionadas por factores psicológicos (usabilidad y utilidad) y sociales (influencia social, apoyo social, condiciones que facilitan el uso, beneficios de su aplicación, etc.), considerando variables moderadoras, la edad, la educación y el tamaño y sector de sus negocios. Para responder a estos objetivos de investigación partimos, como señalamos en el apartado anterior, del Unified Theory of Acceptance and Use of Technology (UTAUT). Al mismo tiempo, intentamos contribuir al avance del modelo realizando un estudio de carácter cualitativo. 
Objetivos específicos:

- Mostrar cómo la facilidad de uso de las RSV es un elemento de atracción y estímulo para iniciarse en la inmersión de la comunicación digital.

- Evidenciar cómo el apoyo social recibido refuerza los sentimientos de inclusión, conexión y pertenencia de la red de la que forman parte (offline y online), además de constituirse en elemento motivador en su proceso de aprendizaje.

- Desvelar qué percepción tienen las mujeres emprendedoras sobre los beneficios/perjuicios potenciales que ofrecen los social media como instrumentos de sus empresas.

- Objetivar las facilidades/dificultades potenciales a las que se enfrentan en la implantación del software social en sus negocios.

El universo está definido por empresarias, es decir, mujeres ocupadas por cuenta propia, autónomas -con y sin asalariados-, titulares de microempre$\operatorname{sas}^{2}$ y pymes. Es decir, personas físicas que realizan habitualmente una actividad económica o profesional a título lucrativo, con residencia y domicilio fiscal en Andalucía, y dan o no ocupación a trabajadoras/es por cuenta ajena y cuya plantilla es inferior, como media, a diez personas asalariadas.

En lo referente a la muestra, la primera fase del proceso de selección comenzó con la elaboración de un marco muestral en el que se registraron empresas andaluzas dirigidas por mujeres. A partir de aquí, la selección se efectuó combinando dos tipos de muestreo cualitativo: muestreo por cuotas (sector empresarial; tamaño de la empresa y autónomas) y muestreo de casos con máxima variación, de forma que aquélla contuviese características geográficas (representatividad de la comunidad andaluza) y sociodemográficas diversas (estudios superiores, estudios básicos o de grado medio; mujeres jóvenes y mayores; con cargas familiares y sin cargas). La muestra está configurada por catorce «informantes» que accedieron a ser entrevistadas.

2. A excepción de una empresa grande de 2000 empleados perteneciente al sector tecnológico.

Feminismo/s 27, junio 2016, pp. 219-242 
Tabla 1. Características de la muestra

\begin{tabular}{|c|c|c|c|c|c|c|}
\hline 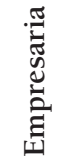 & 莺 & 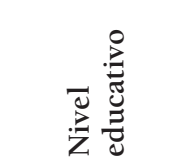 & 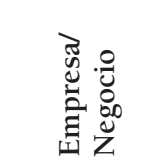 & 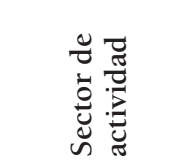 & 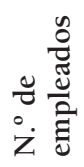 & 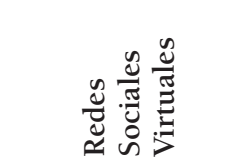 \\
\hline E1 & 36 & CFGS & Autónoma & $\begin{array}{l}\text { Comercio. } \\
\text { Minorista }\end{array}$ & 0 & $\begin{array}{l}\text { Facebook, } \\
\text { WhatsApp }\end{array}$ \\
\hline E2 & 37 & Universitario & Autónoma & $\begin{array}{l}\text { Servicios. } \\
\text { Gestoría }\end{array}$ & 1 & $\begin{array}{l}\text { Facebook, } \\
\text { WhatsApp }\end{array}$ \\
\hline E3 & 38 & Universitario & Autónoma & $\begin{array}{l}\text { Servicios } \\
\text { Consultora }\end{array}$ & 2 & $\begin{array}{c}\text { Facebook, } \\
\text { Twitter LinkedIn, } \\
\text { WhatsApp }\end{array}$ \\
\hline E4 & 38 & Universitario & Autónoma & $\begin{array}{l}\text { Servicios. } \\
\text { Asesoría }\end{array}$ & 1 & $\begin{array}{c}\text { Facebook, } \\
\text { Twitter LinkedIn, } \\
\text { WhatsApp }\end{array}$ \\
\hline E5 & 41 & Universitario & Autónoma & $\begin{array}{l}\text { Servicios. } \\
\text { Educación }\end{array}$ & 1 & $\begin{array}{l}\text { Facebook, } \\
\text { WhatsApp }\end{array}$ \\
\hline E6 & 43 & Universitario & Empresaria & $\begin{array}{c}\text { Servicios } \\
\text { Ambientales }\end{array}$ & 120 & $\begin{array}{l}\text { Facebook, } \\
\text { WhatsApp }\end{array}$ \\
\hline E7 & 48 & Universitario & Empresaria & $\begin{array}{l}\text { Servicios } \\
\text { Turismo }\end{array}$ & 3 & $\begin{array}{c}\text { Facebook, } \\
\text { Twitter LinkedIn, } \\
\text { WhatsApp }\end{array}$ \\
\hline E8 & 48 & $\begin{array}{l}\text { Estudios } \\
\text { Primarios }\end{array}$ & Autónoma & $\begin{array}{l}\text { Comercio } \\
\text { Minorista }\end{array}$ & 0 & $\begin{array}{c}\text { Facebook, Twitter, } \\
\text { WhatsApp }\end{array}$ \\
\hline E9 & 61 & Universitario & Autónoma & $\begin{array}{c}\text { Servicios } \\
\text { Construcción }\end{array}$ & 0 & $\begin{array}{c}\text { Facebook, Twitter, } \\
\text { Instagram, } \\
\text { Pinterest, } \\
\text { WhatsApp }\end{array}$ \\
\hline E10 & 46 & Universitario & Empresaria & $\begin{array}{l}\text { Industrial/ } \\
\text { tecnológico }\end{array}$ & 2000 & $\begin{array}{c}\text { Facebook, } \\
\text { Twitter, LinkedIn, } \\
\text { WhatsApp }\end{array}$ \\
\hline E11 & 49 & Bachillerato & Empresaria & $\begin{array}{l}\text { Comercio } \\
\text { Muebles }\end{array}$ & 10 & $\begin{array}{l}\text { Facebook, } \\
\text { WhatsApp }\end{array}$ \\
\hline E12 & 47 & CFGM & Empresaria & $\begin{array}{l}\text { Industria. } \\
\text { Aceros Inox. }\end{array}$ & 14 & $\begin{array}{l}\text { Facebook, } \\
\text { WhatsApp, } \\
\text { LinkedIn }\end{array}$ \\
\hline E13 & 41 & Universitario & Empresaria & $\begin{array}{c}\text { Industrial } \\
\text { Alimentación }\end{array}$ & 300 & $\begin{array}{l}\text { Facebook, } \\
\text { WhatsApp, } \\
\text { LinkedIn }\end{array}$ \\
\hline E14 & 29 & CFGM & Empresaria & $\begin{array}{l}\text { Industria } \\
\text { Metalurgia }\end{array}$ & 14 & $\begin{array}{l}\text { Facebook, Tuenti, } \\
\text { LinkedIn }\end{array}$ \\
\hline
\end{tabular}

Fuente: Elaboración propia 


\section{ANÁLISIS E INTERPRETACIÓN}

\subsection{Elementos Motivadores de uso e inmersión del empresariado femenino}

\subsubsection{Experiencia real de fácil uso}

Un aspecto que puede actuar como móvil de uso de las RSV es la «usabilidad». Se trata de un término anglosajón que hace referencia a la facilidad de uso de una aplicación o producto. Jakob Nielsen la definió como el atributo de calidad que mide lo fáciles que son de usar las interfaces Web. Los componentes que miden la usabilidad son: la facilidad de aprendizaje, eficiencia, memorización o cualidad de ser recordado, eficacia (errores) y satisfacción (Nielsen 1).

En el 2008 se analizaron trece casos de emprendedoras españolas en tres de las comunidades de mayor tejido empresarial -Madrid, Barcelona y Sevilla-, detectándose que tenían una trayectoria profesional y académica muy heterogénea cuando iniciaron su negocio. De manera específica las mujeres no tenían una formación en el campo de las TIC, y tanto las que no poseían formación superior como las que sí, habían aprendido su uso mediante cursos o de manera autodidacta (Lamolla).

En efecto, RSV conforman un conjunto de recursos intuitivos y de utilización fácil cuyo uso y aplicación se ha extendido exponencialmente entre la población a base de practicar siguiendo el viejo procedimiento de ensayo acierto-error.

Hombre, empecé por Facebook, y yo no lo veía tampoco tan difícil porque como... estos genios informáticos se calientan tanto la cabeza para que a nosotras no sea tan fácil de entrar, en las redes sociales, las programaciones y demás... Está todo muy sencillo, muy masticado, para que tú llegues directamente. (E.3)

Un valor definitorio de las RSV es su capacidad para difuminar los contornos espacio-temporales entre individuos, haciendo posible el encuentro y el vínculo de personas con lazos consanguíneos, intereses semejantes o, simplemente, valores comunes que están distantes geográficamente. En esta línea, estudios de ámbito internacional demuestran que determinadas acciones como las que suponen mantener contactos familiares y de amistades son realizadas fundamentalmente por mujeres (Clipson, Wilson, DuFrene; Mazman, Usluel).

A nivel personal las uso para hablar con mis amistades, a las cuales hace tiempo que no veo. Viven fuera de Sevilla. Viven... esparcidas por el mundo, vamos a decir... Uso el Facebook para estar en contacto, para recordar 
cumpleaños. Entonces, el tener un contacto tan directo también con esas personas que fueron parte de mi vida hace tiempo, y poder mantener el contacto con ellas, me da mucha satisfacción personal [...]. En general, te permite estar continuamente conectada con las personas que quieres: familia, amigos de siempre del instituto, nuevos... (E.9)

Respecto a las diferencias de género en el uso de las redes sociales se han publicado gran cantidad de investigaciones en el ámbito internacional y nacional, aunque sus resultados no son concluyentes variando de unas redes a otras y de unas edades a otras. Thompson y Lougheed demostraron que las mujeres pasan más tiempo en Facebook que los hombres. Por contra, en un análisis exploratorio realizado en España sobre lo que comparten los jóvenes universitarios en Facebook demostró que la brecha de género no es apenas perceptible (Martínez Valerio). Otra investigación sí encontró diferencias, aunque las mismas eran más intensas en relación a la edad, formación y clase social (García, López de Ayala, García).

El Informe «Desarrollo empresarial y redes sociales» indica que suele ser habitual que las microempresas no tengan una cuenta específicamente de uso profesional, sino que realizan un uso profesional y personal (compartido) de las cuentas en las redes sociales (Fundación Telefónica 10). En nuestra investigación, pese a que una minoría así lo haga, en su mayoría las entrevistadas hacen un uso distinto de las mismas.

Yo tengo un perfil personal para las redes sociales, un perfil profesional y un perfil de la empresa que hemos montado. Yo sé perfectamente cuándo estoy en uno o cuándo estoy en otro [...] perfiles, en función de lo que quiera porque además, entiendo que tú no puedas mezclar un comentario personal, con un comentario de negocio, o con un comentario que tú haces de otro negocio. (E.6)

Hay una esforzada y creciente reacción sistemática de determinados colectivos de mujeres por utilizar el potencial de desarrollo de las TIC, y en concreto de las plataformas de comunicación social. Una encuesta realizada en 2011 por el Instituto de Investigación de la Vida de la pequeña empresa reveló que las empresarias tienen tres veces más probabilidades de participar en las redes sociales que sus homólogos varones. En efecto, en Estados Unidos, casi la mitad de las empresarias planificaban incrementar el uso de redes en sus negocios, frente a los empresarios que tenían una propensión mayor a invertir en personal y en formación que en medios de comunicación social (Lugaye et al 554). Pero, además, la Web 2.0 tiene un efecto adicional y de especial calado para el empresariado femenino que se traduce en gestionar y compatibilizar 
un activo muy valioso, como es su tiempo, en los ámbitos familiar y de negocio (Lugaye et al. 558).

Vamos para mi es necesario. Yo no tengo que estar obligada a estar aquí, o sea, yo puedo estar en la mesa con uno de mis hijos haciendo los deberes y yo mis deberes también me los puedo llevar al lado. Y... ¡ hombre!, los límites como acabamos de comentar también tienes que ponerlos, pero sí que es verdad que me queda la tranquilidad de que un domingo por la tarde, yo me anticipo viendo cosas que me voy a encontrar el lunes, porque además llego la última porque mi conciliación familiar la he marcado yo, y el negocio sabe hasta dónde llego. (E.2)

Es conocida la complejidad de papeles que desempeñan estas mujeres emprendedoras en su vida cotidiana: procreadoras, administradoras de casas y dirigentes de sus propios negocios. Las RSV están facilitando la conciliación de su vida personal y laboral.

\subsubsection{Apoyo social informal. Círculos de amistades}

Entendemos por «apoyo social» un conjunto de transacciones interpersonales que suponen ayuda, afecto y afirmación (Barrón 15-17), es decir la forma de asistencia que una persona puede recibir proveniente de sus redes sociales -conjunto de relaciones-. Dicho conjunto de transacciones o «transferencias» se presentan como flujos de intercambios y circulación de recursos, acciones e información. Aquí las redes actúan como marcos donde los intercambios hacen posible la existencia, percepción y recepción de apoyo social.

La iniciación en el uso de las RSV es bastante similar por parte del empresariado femenino. Casi la totalidad empieza con una cuenta personal: por invitación, seducidas por amistades y personas cercanas o, bien, por simple curiosidad; por el hecho de haber oído hablar de ellas en entornos próximos. Algunas investigaciones han puesto de relieve que el sentido de pertenencia a los grupos -en el caso de las mujeres- y las fuertes relaciones recibidas en las redes sociales de apoyo procuran una disposición favorable para persistir en su aprendizaje (Prins, Wilson, Schafft). Estamos ante un uso facilitado y potenciado por «contagio», por «imitación», por «pertenencia».

Que alguien empezaría a hablar del tema «oye pues no sé qué. Pues, yo he estado hablando por el Facebook, pues no sé cuánto. Pues, métete, ábrete la cuenta. Yo es que soy muy poco dada muchas veces a eso». [...] Pues sería algo así también, sería algo de que mis amigas, pues, métete por ahí... (E.8)

Las comunidades virtuales permiten que sus usuarios crean y mantengan vivos los contactos sociales ya iniciados en entornos offline, y contacten asimismo con otras personas con intereses y objetivos comunes (Boyd, Ellison; Ellison, 
Steinfield, Lampe). El apoyo recibido (o percepción de la red social online como una fuente de ayuda), de hecho, se asociará positivamente con crecientes niveles de satisfacción y confianza, mediando estas la decisión del usuario de participar activamente y comprometerse afectivamente. Además, entre los usos de la Web 2.0 se han evidenciado motivaciones diversas: relacionarse socialmente (amigos, familiares), intercambiar información o, acciones compartidas referidas al ocio y entretenimiento (Dogruer, Menevis, Eyyam). De esta manera el apoyo social recibido de la red social offline vuelve a revertirse, reforzarse e intensificarse, digitalmente.

\subsubsection{Inmersión en las redes (frecuencia de uso)}

Nuestro interés se focaliza en conocer la inclusión y grado de inmersión de los social media en la actividad diaria de las empresarias como instrumentos de empoderamiento profesional. En efecto, asumimos que el éxito de dichas plataformas deriva de la participación duradera y presencia activa de las empresas frente a la aceptación inicial. Como dato orientativo a nivel general, según el Observatorio sobre el uso de las Redes Sociales en las Pymes Españolas «el $45 \%$ de las pymes aún no utilizan las plataformas sociales en su día a día corporativo. De estas, el 44\% tiene intención de hacerlo en el futuro» (Fundación Banesto 17).

Lo tengo metido dentro de una rutina, porque, claro, para mí es un trabajo. ¿Eh? tanto la parte personal como la parte: $\mathrm{mmm}$ profesional, que aunque parezca que las mezclo, no las mezclo nunca, pero sí que tengo una rutina. (E.10)

Además, su uso es consuetudinario, forma parte de sus hábitos, de su asiduidad, de su rutina, considerándose la «rutinización» una medida de éxito en las redes sociales. En efecto, la asiduidad del uso o uso cotidiano apoya el proceso de aprendizaje, genera experiencia y conocimiento. La rutinización es la fase en la que se estandariza el uso de la tecnología como apoyo a las tareas sustantivas de un negocio (Fuentes 2).

Pues, estoy demasiado enganchada. Yo creo que me tengo que desenganchar un poco porque me veo yo. Mi marido ya por las noches -nosotros siempre acostamos a los niños y tal... y tenemos un rato- de once y media a doce cada uno lee su libro y, últimamente no tengo libro, sino móvil. Y entonces mi marido empieza a mirarme y empieza « ¿ya no lees?» [... ] Me dice: «¿no vas a coger el libro?» Y yo: sí, sí. «Deja ya el móvil y coge el libro ¿no?» [...] Y por la noche, no sé qué miro, miro mucho el móvil... Entonces, cuando miro el móvil, sistemáticamente repaso el Linkedin, repaso el Facebook y repaso el Twitter. (E.7) 
Entre las entrevistadas el grado de inmersión en las redes no es uniforme: pese haber constatado una correlación menor en algunas titulares de pymes y trabajadoras por cuenta propia, no podría establecerse una relación lineal, al existir otras variables de diferente naturaleza (sector de actividad, volumen de negocio, edad, nivel educativo) que interfieren en su implantación y frecuencia de uso.

\subsection{Eficacia percibida}

\subsubsection{Beneficios/Rendimientos}

Las RSV pueden llegar a ser muy beneficiosas en diferentes ámbitos de marketing y gestión empresarial. Son muchos los usos que las empresas pueden dar a las redes sociales:

El Twitter, el Twitter me aporta, mmm... pues tres cosas: una muy importante el estar al día de la actualidad en on-line; cosa que es muy interesante cuando viajas mucho, porque cuando tú te vas de España pues aterrizas en otro país, enciendes el Twitter y rápidamente te enteras de lo que ha pasado en los veinte primeros tweets, siguiendo los periódicos y siguiendo las cadenas de televisión española, sabes lo que ha ocurrido. Es un periódico ambulante. Luego, lo segundo y muy interesante, información de tu empresa, o sea, buscando lo que se dice de tu empresa. A nivel del mundo, quién menciona a tu empresa, en qué medios de comunicación ha salido, en qué foro está, [...], o sea, temas relacionados con tu negocio; y, en tercer lugar, a nivel de empleados es muy interesante porque la gente de la empresa también habla, menciona a la empresa y también puede tener temas laborales, de gente que esté contenta o descontenta. También puedes evaluar un poco el clima laboral desde el punto de vista interno. Con lo cual el Twitter está claro como herramienta de trabajo. (E.10)

Sin embargo, estaríamos ante un error al creer que lo que motiva el uso de un producto, en este caso las RSV es, exclusivamente, la facilidad de uso. En efecto, en general los usuarios no buscan sólo usabilidad sino también utilidad entendida como provecho, beneficio e interés que produce su uso (Hassan-Montero 241-242). Ahora bien, la relación entre usabilidad y utilidad es dependiente (Dillon). En este sentido podemos considerar que la utilidad que comienza a darse a los social media en el ámbito del mercado, está suponiendo una nueva revolución de la cultura emprendedora digital moderna, dentro de la cual el empresariado femenino tiene todavía un importante reto de inmersión.

Es que si no estás...Yo tengo un amigo informático que me dijo: «oye he visto que tenéis un perfil en Facebook. Estáis montando la empresa, ¿verdad?». Y 
yo le dije, «bueno, ahora la estamos construyendo». Y él me dijo: «Bueno, pero si estáis en Facebook, existís». (E.5)

El mundo empresarial está utilizando el software social para transformar las prácticas de negocios y reinventar la dirección. Algunas empresas utilizan las herramientas de la Web 2.0 para cambiar la gestión y control de las organizaciones a nivel interno. Estas herramientas se usan para mejorar la comunicación entre empleados, o hacer que la gestión de procesos, o la planificación interna sean tareas más sencillas. Asimismo, los departamentos de recursos humanos navegan en ellas en la búsqueda de perfiles que se ajusten a los puestos que ofrecen y necesitan cubrir.

El LinkedIn es vital para conocer gente, para contratar gente nueva, información sobre perfiles $\mathrm{o}$, vas a hacer un negocio con alguien pues rápidamente, en cinco minutos ya sabes quién es, dónde ha trabajado. Entonces, yo el LinkedIn lo veo como una base de datos enorme de currículum y también a nivel internacional, o quieres conectar con un cliente nuevo, o es un método de acercamiento comercial, ¿no? (E.10)

En este sentido, los social media facilitan la diseminación de la información e innovación, además de impulsar las redes de contacto personales, factor fundamental en las empresas pequeñas (pyme) y, particularmente, importante para las emprendedoras (Hill, Scott).

La nueva ola digital y social ha roto el modelo de distribución de contenido centralizado y ha situado en su matriz al consumidor como creador. En efecto, debido a la naturaleza de las redes digitales es posible acercarse al público objetivo con mayor facilidad. Son un canal de difusión, además de representar una fuente de información de primera mano para las empresas, ya que en ellas es posible descubrir necesidades o preocupaciones de los individuos que allí interactúan.

Las redes sociales me ahorran mucho tiempo, porque no solo es que te enteras de lo que piensa uno, de lo que piensa el otro y te enriqueces porque todo va en su versión y tú ya logras hacer la tuya. (E.2)

Además la creación de contenidos, su publicación y que estos sean compartidos genera una difusión a mayor escala, lo que favorece que las empresas estén mejor posicionadas entre los buscadores. De igual modo, las redes facilitan el conocimiento del producto ofertado, por ello es necesario pedir permanentemente la opinión de los usuarios. 


\subsubsection{Perjuicios}

El aprovechamiento de las redes sociales online tiene aspectos positivos para la gestión comunicativa de las empresas como acaban de reconocer sus directivas, sin embargo hacen referencia también a los efectos perversos:

Hay que estar, no, pero, las redes sociales son un arma de doble filo. Hay que ser muy cuidadosa porque, igualmente te pueden dar buen resultado, buena publicidad, que como te den mala publicidad $\mathrm{mmm}$, te la cargas bien. Te la cargas muy bien porque las redes sociales van así. No te da tiempo a dar un chasquido cuando ya se ha difundido el mensaje negativo por toda la red, y es incontrolable. Hay que tener mucho cuidado con el tema de las redes sociales. (E.14)

Las empresas pueden competir en dos mundos no excluyentes, sí complementarios, y en cierta medida continuos: el mundo real de un mercado físico y el mundo virtual donde bienes y servicios pertenecen a un mercado digital. Aquí se da un tipo de comunicación ágil y directa que ha pasado de ser unidireccional a una comunicación participativa. Así, las redes sociales, además de ser un canal de difusión, tienen la bondad/ perversidad de ser un canal de retroalimentación a través del análisis de los comentarios de los usuarios. Los consumidores confían en la información disponible en las redes antes de consumir un producto o servicio, para después compartir.

Es curioso porque hay gente que de repente no son consumidores pero se encuentran con algo que le ha escrito otro, de rebote, y dice: ¿uf? ¿Y esto qué es? O, esto me interesa. Y claro, es que hemos encontrado un nicho perdido que lo estamos rescatando gracias a eso. (E.13)

En el discurso anterior, la entrevistada hace referencia a la capacidad de viralización de los contenidos que poseen las redes sociales, capaces de multiplicar el efecto «epidemia»-tanto positiva como negativamente- que puede tener para la reputación del negocio.

Qué tengo que priorizar y qué emergencias tengo que atender. Si sé que hay una queja de un cliente que ya la ha publicado y que ha compartido con nosotros, todo eso que también me ocupa un poco de la reputación [...]. Y todo no tiene la misma transcendencia, tú filtras y eres capaz de medir, si esto es importante lo tengo que ver con detalle, si esto es.... (E.12)

En este sentido, imagen y reputación de las empresas son dos de los intangibles más vulnerables en la telecomunicación y conversaciones instantáneas de las plataformas de participación en la red. Ambas, son percepciones públicas sobre una empresa o compañía que configuran una relación gestáltica de figura/fondo. La imagen actúa como figura, y la reputación constituye el fondo (Villafañe 39-46). Si bien tienen conciencia de la incidencia de imagen 
y reputación que procuran estas «conversaciones participativas», existe también entre estas empresarias un pensamiento crítico, reflexivo sobre el alcance viral de estas comunidades virtuales.

Tú no puedes ser una esponja y creerte todo lo que ahí está, ¿vale? lo que está en Facebook existe. No, lo que está en Facebook existe, o no. Es que ya es una alarma social con cualquier cosa que sale en Facebook hay que contrastarlo y entonces en ese aspecto es un obstáculo, una desventaja. (E.7)

Los relatos del empresariado femenino muestran un cierto consenso en torno a conceptos como «prudencia», «sensatez» o, matices tales como, «el cuidado en la forma de lo que escribas» que deben estar presentes en lo que se publica, el cómo se publica, la escucha activa y atenta, etc.

¿El negativo?, pues, que hay que andar con prudencia, como todo en la vida. Hacerlo con sentido común y bueno, como todo, es tu forma de comportarte, fuera de tu trabajo, en tu entorno familiar, cuando te vas a la calle y sales de copas, es: «el saber estar». (E.6)

Conscientes de la controversia tanto a nivel privado como profesional/empresarial, respecto a los beneficios e impactos negativos que intrínsecamente supone la utilización de los social media, el empresariado femenino expresa su aquiescencia sobre un mayor provecho y beneficio en su mundo profesional circundante:

La importancia para mí de que ellos tuvieran este curso (formación de su equipo) era para que se crearan su perfil profesional en Linkedin, para que estuviera todo el equipo, para que entendieran que las redes sociales no es cuestión de un entretenimiento, sino bien enfocado es una herramienta muy importante para crear tu marca, para apoyarla, para, crear valor sobre la actividad que desarrollas. (E.3)

\subsection{Factores contextuales}

4.3.1 Condicionantes: tamaño de las empresas o negocios y escasa formación

En nuestra investigación, descubrimos que las experiencias profesionales con las RSV en el ámbito empresarial podrían ser clasificadas en tres categorías:

- Grupo minoritario de empresarias reactivas. Reconocen la necesidad de utilizar las RSV, pero especialmente su desconocimiento, la falta de competencias, el recelo, etc., hacen que no tengan una cuenta específicamente de uso profesional. En esta categoría se encuadran algunas autónomas, con formación elemental y media, edades más avanzadas 
y con negocios pequeños concentrados en determinados sectores de actividad comercial de corte tradicional.

- Emprendedoras proactivas. Después de haberlas experimentado en su vida personal, los social media pasan al ámbito de negocio. Se trataría de un uso «cuasi-profesional» centrado sobre todo en la comunicación externa: relación con los clientes, promoción de productos o servicios, y venta online. En esta categoría se incluyen mujeres autónomas, titulares de microempresas, con formación universitaria, de amplio espectro de edad -con y sin empleados a su cargo-, y con un interés creciente por realizar mayores acciones.

- Grupo minoritario de empresarias digitales. Con formación específica en redes virtuales y que realizan un uso profesional de estas plataformas, tanto dentro como fuera de sus empresas. En este nivel se encuadran medianas y grandes empresas en sectores de producción en los que la implantación de estas nuevas tecnologías y su gestión por parte de profesionales devienen en indispensables.

Se ha hecho explícita en nuestra investigación, la necesidad del empresariado femenino de avanzar en el nivel formativo generador de competencias digitales al objeto de capacitarse. Aspecto éste clave para la obtención de oportunidades abiertas para la gestión de una cartera de clientes y como potente recurso en la modernización de sus negocios. El siguiente testimonio ilustra la experiencia de una empresaria beneficiada por unas acciones formativas del Instituto de la Mujer (Junta de Andalucía):

Hoy hay muchas empresas dedicadas al posicionamiento, a las tiendas, hay muchos profesionales relacionados con el tema de subir, de hacerte ser visible en Internet, [...], pero, claro, ellos no conocen mi empresa, la que conoce mi empresa soy yo. Yo soy la persona que tiene que saber qué redes sociales le vienen bien a mi empresa, al igual que si me conviene o no tener página de ventas online, el posicionamiento dónde me conviene, o las palabras técnicas para estar bien posicionada. Antes de realizar el curso, pensaba que necesitaba un profesional, que me dijera a mí lo que tenía que hacer con mi empresa en Internet para que fuera visible, pero no, no es así. Soy yo la persona que le tiene que decir al profesional lo que tiene que hacer y dónde se tiene que anunciar para que mi empresa sea visible en Internet. Y, muy importante, cómo yo quiero que vean mi público en Internet. (E.14)

En ciertos casos las competencias adquiridas con el uso personal de las redes se ha complementado con una formación específica sobre el uso y aplicación de estos instrumentos al ámbito empresarial. Aplicar una política en redes 
a los negocios supone una planificación y una estrategia de contenidos que requiere de una labor especializada.

Hice un curso y me encantó. Yo quería gestionar las redes de mi empresa. [...] Y, ya llega un momento en que yo no puedo abarcar, no puedo, y lo tengo que delegar, pero por lo menos, saber. [...] Al hacer este curso me llevé las tablas necesarias para hacerlo, creo, de manera más correcta. (E.6)

\subsubsection{Expectativa de rendimiento y ambiente circundante.}

Las empresarias ratifican la implantación de las RSV, apostando por la mejora de sus negocios, de sus relaciones, contactos, etc., de tal manera que, la razón por la que aquéllas las utilizan vendrá determinada por el cumplimiento y rentabilidad de sus objetivos.

A mí me ahorra mucho tiempo, y estoy siempre actualizada. (E.3)

Me ayuda a que vaya mejor [...] lo vendes a través de Facebook que te ayuda muchísimo. [...] Lo voy a seguir utilizando, porque le veo casi más salidas que en la tienda. (E.1)

La participación de estas empresarias en el software social enfatiza la utilidad y funcionalidad mercantil. En efecto, el Observatorio sobre el uso de las Redes Sociales en las Pymes Españolas (Fundación Banesto 51-60) señala que, las empresas utilizan las redes sociales para aquello en lo que ven que supone una ventaja competitiva clara respecto a sus rivales: la manera en que mejora la competitividad es el hecho de darse a conocer en el mercado y establecer un canal de comunicación directo con sus clientes.

Me comunico con los clientes. Con los clientes finales, con los consumidores. En la empresa está claro que cualquier mini sondeo de mercado y a nivel global, la globalización que ha venido unida también a la conexión a la dos punto cero es, es una cosa inmediata, rápida y fiable, porque es que sabes desde qué punto te están respondiendo del globo. [...] Son capaces de compartir contigo cosas que en realidad no saben lo valioso que son para nosotros, porque es que si supieran lo valioso que es ipedirían comisión! Porque se comparten cosas que tú dices ¿oye? ¡Qué esto tiene un valor! (E.6)

De cara al marketing, si tú no estás en Facebook, no eres nadie. (E.4)

Los social media son vitales para la nueva generación de empresas, para promover una nueva revolución de la cultura emprendedora. Fundadora de Altimeter Group y experta en Redes Sociales, Charlene Li, en una entrevista concedida al diario El País, decía respecto a las redes sociales en las empresas que: «Las que carezcan de este diálogo con los clientes no sobrevivirán, y el 
tiempo de su caída dependerá del entorno y del país en el que se encuentren». (Blázquez 29).

En el mundo de la empresa, en cuanto ves cómo se mueve la competencia, cómo sale esto, cómo sale en Expansión, como tal. Estás o no estás y, yo creo, que más que vanguardista es estar con «el hacia dónde vamos» y, tener un poco de visión, porque en una empresa siempre tienes que tener cierta planificación estratégica, ¿no? (E.10)

La crisis económica entendida como situación sorpresiva e inesperada, obviamente afecta a la estabilidad de los negocios. En alguna propietaria de pymes hemos advertido el miedo y, sobre todo el desconocimiento funcional de las plataformas digitales como instrumentos que podrían coadyuvar a reflotar su negocio:

No somos una empresa modelo en tema de tecnología puesto que, dadas las circunstancias y pidiendo presupuesto como he hecho ya, hace precisamente poco tiempo, para actualizar lo que es la página Web y lo que es el Facebook y demás..., y que nos llevaran el tema, pues, ahora mismo sería inviable porque, económicamente, pues no podemos asumir ese gasto en las circunstancias que estamos. [...] Pero sé realmente que, a mí, que a mi empresa le vendría genial estar presente pero de primera página. [...] Y con eso quiero decir que en ese aspecto la tecnología nos vendría genial, porque nos evitaría muchísimo trabajo también, pero vuelvo otra vez a lo mismo. Yo tengo ahora mismo muchas ganas de bailar pero las manos las tengo atadas... (Alude al momento crítico por el que está pasando su empresa: reducción de plantilla). (E.11)

En general, las empresarias conscientes de la dimensión y oportunidad de las plataformas virtuales para su visibilidad, proyección y competitividad, afirman que el futuro de sus negocios pasa, necesariamente, por una mejor comunicación digital.

Para promocionar el negocio hay que tener proyección y para generar negocio, las redes sociales son un elemento gratis y potente. (E.4)

\section{CONCLUSIONES}

Los hallazgos de esta investigación nos permiten constatar que un segmento del empresariado femenino comienza a apostar por las TIC a través de las plataformas participativas de la Web 2.0. Son potentes, útiles e imprescindibles herramientas de comunicación y gestión de clientes. Se trata de un recurso de capacitación, de empoderamiento de las empresarias en la modernización de sus negocios. Las faculta para un «empuje de fronteras» ante las demandas de un mercado global y competitivo. La trascendencia del uso de las redes 
sociales está especialmente en el cambio cultural que este colectivo está dispuesto a asumir, permitiendo, entre otros, un acercamiento y una comunicación con la clientela real y potencial antes inimaginable.

Inicialmente la presencia de las mujeres en las redes sociales con fines personales y la facilidad de uso, las ha advertido de los rendimientos que podrían tener como instrumentos de comunicación en sus empresas. En efecto, las empresarias, seducidas en su vida personal por estas comunidades virtuales, se topan con un recurso o mecanismo intuitivo y sencillo, capaz de recrear entornos de intercambio que difuminan el marco espacio-temporal de sus encuentros personales: familia, amistades, etc. En su iniciación digital tienen mucho que ver sus grupos de pertenencia y las fuertes relaciones de las redes de apoyo que les van a procurar una predisposición favorable en la persistencia de su aprendizaje. Tales comienzos son la antesala que sirve de acicate para la implantación instrumental de las RSV en sus negocios o empresas. Las asumen por el provecho y utilidad que les reportan y, especialmente, la oportunidad de negocio que suponen. La asiduidad de uso, o lo que es lo mismo, la frecuencia y rutina se convierten en barómetro de su implantación e integración. En su relación con el software social, encuentran un efecto adicional no previsto que les permite márgenes de compatibilidad entre el tiempo doméstico-familiar y el público de sus negocios.

No obstante, el empresariado femenino no presenta homogeneidad en el uso y afectación de los social media. La implantación puede estar limitada, sobre todo en el caso de pequeñas empresas y autónomas a usos básicos. Tamaño y sector pueden actuar como condicionantes para usos más profesionales.

Si bien son conocidas las RSV como recursos que pueden generar un valor añadido en el contexto empresarial, aún no han permeabilizado ni sus beneficios ni un conocimiento funcional. Su implantación puede estar en concomitancia con la rama de actividad y el tamaño de la empresa. No obstante, no hay una relación lineal si interactúa con otras variables como la formación académica y la edad de las empresarias. La Web 2.0 como instrumento del empresariado femenino podría encontrarse en un umbral de desarrollo condicionado por factores personales (edad, formación) y contextuales (sector, tamaño de la empresa). Urge que se implementen y aprendan la utilización de los recursos que precisa la nueva economía digital. Ya lo hacen muchas de nuestras empresarias. Pero no es suficiente. Queda un largo recorrido.

Nuestro estudio exploratorio también indica que algunas de estas mujeres empresarias no han recibido una formación específica en el manejo de las RSV como instrumentos de trabajo para sus empresas, sugiriendo que se 
ha producido sólo en contextos informales y con redes sociales de apoyo. Precisamente entendemos que su proceso formativo a través de las acciones políticas supondría un refuerzo hacia su empoderamiento económico. Es una necesidad apremiante la capacitación digital del empresariado femenino concentrado específicamente en pequeños negocios, sectores concretos y de menor capital. Aunque la Agenda Digital para España contempla ciertas medidas al respecto, las investigaciones más utilizadas por los policy makers soslayan informaciones sobre la variable género. Consecuentemente las decisiones operan de forma recurrente, enfatizando intervenciones en aspectos detectados en el empresariado visible masculino. Nuestro análisis aporta una primera aproximación a esta otra realidad diferencial cualitativa. Su limitación principal está en su circunscripción espacial -contexto andaluz-.

Muy probablemente en un futuro próximo, las mujeres sean el principal pilar de crecimiento. De ahí que el esfuerzo político por capacitar al empresariado femenino redunde en la mejora de la productividad, competitividad y equidad en una renovada relación de empoderamiento entre género y TIC.

\section{REFERENCIAS BIBLIOGRÁFICAS}

Asociación de Trabajadores Autónomos (ATA). Informe Mujer Autónoma 2015. Madrid: Federación Nacional de Asociaciones de Trabajadores Autónomos, 2015.

Barrón, Ana. Apoyo social. Aspectos teóricos y aplicaciones. Madrid: Siglo XXI de España Editores, 1996.

Blázquez, Susana. «La empresa que no dialogue con los clientes morirá». El País, 17 abril 2011:29.

Boyd, Dana M. y Nicole B. Ellison. «Social Network Sites: Definition, History, and Scholarship». Journal of Computer-Mediated Communication 13 (2008): 210-230.

Brynin, Malcolm. «Gender, Technology and Jobs». The British Journal of Sociology 57.3 (2006): 437-453.

Clipson, Timothy W, S. Ann Wilson y Debbie D. DuFrene. «The Social Networking Arena: Battle of the Sexes». Business Communication Quarterly 75 (2012): 64-67.

Davis, Freed D. A technology Acceptance Model for empirically testing new end-user information System: Theory and results. Massachusetts: Massachusetts Institute of Technology, 1986.

Davis, Freed D. «Perceived usefulness, perceived ease of use, and user acceptance of information technology». MIS Quarterly 13.3 (1989): 319-340. 
Dillon, Andrew. «Beyond Usability: Process, Outcome and Affect in human computer interactions». Canadian Journal of Information Science 26. 4 (2001): 57-69.

DiMaggio, Paul, Eszter Hargittai, Coral Celeste y Steven Shafer. «Digital Inequality: From Unequal Access Todifferentiated Use». Social Inequality. Ed. Kathryn Neckerman. NewYork: Russell Sage Foundation, 2004, 355-400.

Dogruer, Nazan, Ipek Menevis y Ramadan Eyyam. «What is the motivation for using Facebook?». Procedia Social and Behavioral Sciences 15 (2011): 2642-2646.

Ellison, Nicole B., Charles Steinfield y Cliff Lampe. «The Benefits of Facebook 'Friends': Social Capital and College Students Use of Online Social Network Sites». Journal of Computer-Mediated Communication 12 (2007): 1143-1168.

European Commission. «Digital Agenda for Europe». European Union, 2010. <http://europa.eu/pol/pdf/flipbook/en/digital_agenda_en.pdf>, consultado el 12-06-2014.

Fishbein, Martin e Icek Ajzen. Belief, Attitude, Intention and Behavior: An Introduction to Theory and Research. Reading, MA: Addison-Wesley Pub. Co., 1975.

Fuentes de Iturbe, Patricia. «La adopción tecnológica y sus determinantes». Observatorio para la cibersociedad, 2006. <http://eprints.rclis.org/7634/1/ adopcion7.pdf>, consultado el 12-02-2015.

Fundación Banesto (ed.). Observatorio sobre el uso de las Redes sociales en las Pymes Españolas. Madrid: Fundación Banesto, 2013.

Fundación Iniciador y Sage España. Observatorio de Clima Emprendedor 2012. Un estudio sobre el emprendimiento en España, 2012. <http://www.sodercan. es/wp-content/uploads/sites/4/2015/06/10-Observatorio_Clima_Emprendedor2012.pdf>, consultado el 23-06-2014.

Fundación Telefónica. Desarrollo empresarial y redes sociales El caso de las microempresas españolas. Barcelona: Ariel, 2014.

García, Antonio, María Cruz López de Ayala y Beatriz Catalina García. «Hábitos de uso en Internet y en las redes sociales de los adolescentes españoles». Comunicar 41 (2013): 195-204.

Hargittai, Eszter y Gina Walejko. «The participation divide. Content creation and sharing in the digital age 1». Information, Communication E Society 11.2 (2008): 239-256.

Hassan-Montero, Yusef. «Factores del Diseño Web Orientado a la Satisfacción y No-Frustración de Uso». Revista Española de Documentación Científica 29. 2 (2006): 239-257.

Hill, Jimmy y Terri Scott. «A consideration of the roles of business intelligence and e-business in management and marketing decision making in knowledgebased and high-tech start-ups». Qualitative Market Research: An International Journal 7.1 (2004): 48-57. 
Korupp, Sylvia y Marc Szydlik. «Causes and Trends of the Digital Divide». European Sociological Review 21. 4 (2005): 409-422.

Lamolla, Laura. «Las mujeres en el sector TIC». Género y TIC. Presencia, posición y políticas. Ed. Cecilia Castaño. Barcelona: UOC Ediciones, 2010, 291-320.

Lee, Younghwa, Kenneth A. Kozar y Kai R. T. Larsen. «The technology acceptance model: Past, present, and future». Communications of the Association for Information Systems 12.50 (2003): 752-780.

Livingstone, Sonia y Ellen Helsper. «Gradations in digital inclusion: children, young people and the digital divide». New Media E Society 9.4 (2007): 671-696.

Losh, Susan. «How Educational Level Influences The Gender Digital Chasm in Computer and Internet Access and Use: 1983-2000». IT E Society 1.4 (2003): 73-86.

Lugaye, Celestine, Andre D. Slabbert y Wilfred I. Ukpere. «Rising Trend in Social Media Usage by Women Entrepreneurs across the Globe to Unlock Their Potentials for Business Success». Mediterranean Journal of Social Sciences MCSER 5.10 (2014): 551-559.

Martínez Valerio, Lizette. «Facebook y los jóvenes universitarios: qué comunican según el género». Historia y Comunicación Social 18. N. ${ }^{\circ}$ Especial Diciembre (2013): 77-87.

Mazman, S. Guzin y Yasemin Kocak Usluel (2011). «Gender Differences in Using Social Networks». TOJET: The Turkish Online Journal of Educational Technology 10.2 (2011): 133-139.

Ministerio de Industria, Energía y Turismo. Agenda Digital para España, 2013. <http://www.agendadigital.gob.es/agenda-digital/Paginas/agenda-digital. aspx>, consultado el 12-06-2014.

Nielsen, Jakob. «Usability 101: Introduction to usability». Nielsen Norman Group, 2012. <https://www.nngroup.com/articles/usability-101-introduction-to-usability/>, consultado el 12-02-2015.

Norris, Pippa. Digital divide. Civic engagement, information poverty and the Internet world wide. Cambridge, Massachussets: Cambridge University Press, 2001.

Prins, Esther, Blaire Wilson y Kai A. Schafft. «It Feels Like a Little Family to Me: Social Interaction and Support among Women in Adult Education and Family Literacy». Adult Education Quarterly 59.4 (2009): 335-352.

Rodríguez-Díaz, Rosario, María José González-Río y M. a Ángeles Rebollo. «Mujeres empresarias en el uso de la web 2.0. Discursos de un preludio». Revista Internacional de Ciencias Sociales Interdisciplinares 3. 2 (2014): 11-25.

Thompson, Sharon. H. y Eric Lougheed. «Frazzled by Facebook? An exploratory study of gender differences in social network communication among undergraduate men and women». College Student Journal 46.1 (2012): 88-98. 
Torres, Cristóbal, José Manuel Robles y Oscar Molina. «¿Por qué usamos las tecnologías de la información y las comunicaciones? Un estudio sobre las bases sociales de la utilidad de Internet». Revista Internacional de Sociología 69.2 (2011): 371-392.

Venkatesh, Viswanath y Freed D. Davis. «A Theoretical Extension of the Technology Acceptance Model: For Longitudinal Field Studies». Management Science 46. 2 (2000): 186-204.

Venkatesh, Viswanath, Michael G. Morris, Gordon B. Davis y Freed D. Davis. «User Acceptance of Information Technology a Unified View». MIS Quarterly 27.3 (2003): 425-478.

Venkatesh, Viswanath y Hillol Bala. «Technology Acceptance Model 3 and a Research Agenda on Interventions». Decision Sciences, 39.2 (2008): 273-315. Villafañe, Justo. La buena reputación. Madrid: Pirámide, 2004. 